\title{
Reanimação da face paralisada: 0 ponto de vista neurocirúrgico
}

\author{
Marcus André Acioly', Carlos Telles², Pedro Santana ${ }^{3}$, Paulo Henrique Pires de Aguiar ${ }^{4}$ \\ Disciplina de Neurocirurgia da Universidade do Estado do Rio de Janeiro \\ Disciplina de Neurocirurgia da Faculdade de Medicina da Universidade de São Paulo
}

\section{RESUMO}

Objetivo: Revisão e discussão de técnicas consagradas na reanimação da face paralisada e das inovações sobre o tema. Método: Pesquisa bibliográfica abrangendo o banco de dados eletrônico Medline e pesquisa manual em jornais e revistas médicas, compreendendo o período de 1980 a 2008; facial nerve palsy, facial paralysis, reanimation e reconstruction foram utilizadas como palavras-chave. Conclusão: A despeito dos avanços nas técnicas de monitorização intraoperatória, a lesão do nervo facial permanece sendo uma complicação frequente nos pacientes submetidos à cirurgia de base de crânio. A paralisia facial acarreta alterações funcionais, cosméticas e psicológicas, representando um grande desafio à cirurgia reconstrutiva. Atualmente, uma miríade de técnicas encontra-se disponível para reanimação da face paralisada. A escolha da abordagem adequada leva em consideração a idade do paciente, o tempo de lesão facial, assim como a topografia da lesão do nervo facial, entre outros.

\section{PALAVRAS-CHAVE}

Paralisia facial, reabilitação. Paralisia facial, cirurgia.

\section{ABSTRACT}

Reanimation of the paralyzed face: the neurosurgical point of view

Objective: To review and discuss emphasizing the common surgical techniques and the state-of-the-art techniques for paralyzed face. Method: Bibliographic search in Medline and manual review of related publications from 1280 to 2008; the searching words were: facial nerve palsy, facial paralysis, reanimation e reconstruction. Conclusion: Despite recent advances in intraoperative monitoring, facial nerve lesion still is a complication of major concern in patients undergoing skull base surgeries. Facial nerve palsy has functional, cosmetic and psychological impact on patients representing a challenge to reconstructive surgery. Currently a myriad of surgical techniques is available for reanimation of the paralyzed face. The surgical management for reanimation considers not solely the patient's age, but also duration of facial palsy and topography of facial nerve injury, among others.

\section{KEY WORDS}

Facial paralysis, rehabilitation. Facial paralysis, surgery.

\section{Introdução}

A preservação da função do nervo facial é um dos maiores desafios ao cirurgião de base de crânio ${ }^{11}$ e de cabeça e pescoço. A despeito dos avanços nas técnicas de monitorização intraoperatória, a lesão do nervo facial ainda é uma complicação frequente nos pacientes submetidos à cirurgia de base de crânio. ${ }^{11,16,20} \mathrm{~A}$ paralisia do nervo facial que sobrevém é uma das lesões de nervos periféricos mais incapacitantes, tendo em vista as alterações funcionais, cosméticas e psicológicas que acarreta. ${ }^{2,10,18,21}$

Atualmente existe uma grande variedade de técnicas dinâmicas e estáticas para a reanimação da face paralisada. ${ }^{10,11}$ No entanto, nenhuma delas é capaz de restaurar completamente a função facial. ${ }^{10}$ Embora os movimentos voluntários sejam recuperados, o restabelecimento dos movimentos involuntários espontâneos perdidos associados à expressão das emoções não ocorre com nenhum método disponível no momento. ${ }^{10,21}$

1 Professor substituto da disciplina de Neurocirurgia, Hospital Universitário Pedro Ernesto, Universidade do Estado do Rio de Janeiro, fellow do Departamento de Neurocirurgia do Hospital Universitário de Tübingen, Alemanha.

2 Professor adjunto e chefe da disciplina de Neurocirurgia, Hospital Universitário Pedro Ernesto, Universidade do Estado do Rio de Janeiro. 3 Neurocirurgião assistente, disciplina de Neurocirurgia, Departamento de Neurologia, Faculdade de Medicina da Universidade de São Paulo. 4 Professor livre-docente, disciplina de Neurocirurgia, Departamento de Neurologia, Faculdade de Medicina da Universidade de São Paulo. 
A escolha da técnica apropriada deve incluir a topografia e a etiologia da lesão do nervo facial, a idade do paciente, a extensão e a gravidade dos déficits cosméticos associados, o tempo de lesão, o estado geral do paciente, a doença de base, o planejamento terapêutico futuro e os objetivos pessoais do paciente. . $^{718,21}$

O resultado ideal da reanimação da face paralisada inclui a restauração completa do tônus muscular, a simetria da face no repouso e durante os movimentos faciais voluntários, bem como ausência de sincinesias. ${ }^{10}$ No entanto, o objetivo primordial é restabelecer a função do esfíncter do olho para proteção da córnea ${ }^{10,13} \mathrm{e}$ a competência do esfíncter oral. ${ }^{10}$

Esta revisão objetiva a discussão de técnicas consagradas na reanimação da face paralisada e das inovações sobre o tema.

\section{Método}

Uma pesquisa bibliográfica abrangendo o banco de dados eletrônico Medline e pesquisa manual em jornais e revistas médicas, compreendendo o período de 1980 a 2008, foi realizada. Facial nerve palsy, facial paralysis, reanimation e reconstruction foram utilizadas como palavras-chave. Didaticamente, discutimos as técnicas em três categorias de procedimentos, divididos de acordo com a topografia da lesão do nervo facial, a saber: perda da continuidade intracraniana com coto proximal disponível, perda da continuidade intracraniana com coto proximal indisponível ou não funcional e perda da continuidade extracraniana (Quadro 1).

\section{Perda da continuidade intracraniana com coto proximal disponível}

O processo de crescimento dos tumores de fossa posterior causa um alongamento do nervo facial e, eventualmente, infiltração deste de forma crônica. ${ }^{15}$ Assim, durante a cirurgia a continuidade do nervo facial no ângulo ponto-cerebelar (APC) não pode ser preservada em $5 \%$ a $10 \%$ desses tumores. ${ }^{15}$ Nesses casos, a reconstrução imediata intracraniana deve ser considerada..$^{15}$

A restauração da continuidade do nervo facial é a única forma de restabelecer de forma aproximada o equilíbrio normal da face. ${ }^{7}$ A porção intracraniana do nervo facial pode ser reparada no APC ou no osso petroso quando o coto proximal estiver disponível. ${ }^{7,15,16}$ O reparo primário promove os melhores resultados funcionais por meio da anastomose término-terminal direta ou com interposição de enxerto nervoso, nos casos em que a distância entre os cotos não permita síntese direta sem tensão., ${ }^{2,10,11}$ Corriqueiramente, os nervos auricular magno e sural são os mais utilizados para enxertia nervosa. ${ }^{7} \mathrm{O}$ nervo auricular magno pode ser utilizado para enxertos curtos de até $8 \mathrm{~cm}$, e o nervo sural, para enxertos mais longos de até $12 \mathrm{~cm}^{7}$

A anastomose término-terminal tende a apresentar uma recuperação mais precoce em relação à interposição de enxertos, embora essa diferença não seja estatisticamente significativa. ${ }^{10}$ A recuperação pode ser evidenciada no sexto mês de pós-operatório em 54,3\% dos pacientes submetidos à anastomose término-terminal, sendo a melhora documentada de forma contínua, mesmo após 24 meses do procedimento. ${ }^{10}$ Resultados satisfatórios, leia-se paciente com classificação funcional de House-Brackmann (HB) para o nervo facial menor ou igual a III, são alcançados em 51\% a 84,6\% dos casos. ${ }^{7,10,15,16}$ Sendo os melhores resultados atingidos nos pacientes em que a anastomose término-terminal foi realizada. ${ }^{10} \mathrm{O}$ músculo frontal recupera a função em até $41 \%$ dos casos. ${ }^{15}$

Em casos selecionados, como nos tumores do osso petroso, procedimentos alternativos de interposição de enxertos intracraniano-intratemporal ou intraextracraniano podem ser considerados com resultados satisfatórios. ${ }^{15,16}$ Recentemente, uma nova técnica de

\begin{tabular}{|c|c|}
\hline \multicolumn{2}{|c|}{$\begin{array}{c}\text { Quadro } 1 \\
\text { Técnicas atualmente disponíveis para reanimação da face paralisada de acordo com a topografia da lesão do nervo facial. }\end{array}$} \\
\hline Topografia da lesão & Técnica \\
\hline $\begin{array}{l}\text { Perda da continuidade intracraniana } \\
\text { (coto proximal disponível) }\end{array}$ & $\begin{array}{l}\text { Reparo direto (anastomose TT). } \\
\text { Reparo com interposição de enxertos (anastomose TT ou TL). }\end{array}$ \\
\hline $\begin{array}{l}\text { Perda da continuidade intracraniana } \\
\text { (coto proximal indisponível ou não funcional) }\end{array}$ & $\begin{array}{l}\text { Anastomose hipoglossofacial clássica. } \\
\text { Anastomose hipoglossofacial com interposição de enxerto (jump graft). } \\
\text { Anastomose hipoglossofacial intrapetrosa TL. }\end{array}$ \\
\hline Perda da continuidade extracraniana & $\begin{array}{l}\text { Reparo direto periférico ou com enxerto. } \\
\text { Transferência nervosa transfacial (cross-facial nerve graft). } \\
\text { Transferência muscular microneurovascular. } \\
\text { Transferência muscular regional. } \\
\text { Procedimentos estáticos de suporte. }\end{array}$ \\
\hline
\end{tabular}

$\mathrm{TT}=$ término-terminal; $\mathrm{TL}=$ término-lateral 
interposição de enxertos foi descrita para reconstrução do nervo facial, chamada de interposição términolateral. ${ }^{14}$ Essa técnica foi utilizada seguindo-se à ressecção de um schwannoma vestibular (tamanho T4b de Hannover), em que apenas 30\% das fibras do nervo facial estavam preservadas ao final da cirurgia, e um bloqueio funcional foi observado como resposta à estimulação direta do nervo no APC. ${ }^{14} \mathrm{~A}$ interposição término-lateral do enxerto de nervo sural foi feita com o intuito de reforçar a função dos fascículos preservados. O paciente apresentou recuperação facial funcional HB III 18 meses após o procedimento. ${ }^{14}$

\section{Perda da continuidade intracraniana com coto proximal indisponível ou não funcional}

Existem quatro situações em que o nervo facial não pode ser reconstruído por intermédio de reparo direto ou enxerto, quais sejam: no caso de perda do coto proximal do nervo; no caso de lesão do núcleo do nervo facial; nos casos de ausência de recuperação funcional do nervo com preservação anatômica deste; e nos casos de ausência de recuperação funcional do nervo seguindo-se ao reparo direto ou enxerto de nervo intracraniano. ${ }^{10,15}$

Quando existe descontinuidade do nervo facial no APC, a reanimação imediata ou precoce deve ser feita ${ }^{16}$ sendo geralmente necessária a translocação de outro nervo craniano, mais comumente o nervo hipoglosso. ${ }^{2,4,11,16,17}$ No entanto, naqueles pacientes em que houve preservação anatômica do nervo funcional no APC devem-se aguardar 12 meses para observar alguma recuperação funcional antes da indicação cirúrgica. ${ }^{7,16}$ A mesma conduta expectante é considerada nos pacientes submetidos ao reparo direto ou à interposição de enxertos no APC, de forma que um período de $12 \mathrm{ou}$ 24 meses é necessário para observação da recuperação funcional, respectivamente. ${ }^{10}$ Tempo é a palavra-chave para a utilização da técnica de anastomose hipoglossofacial (AHF). ${ }^{11,18,21}$ Nas lesões do nervo facial ocorridas há dois ou até quatro anos, a AHF ainda pode ser realizada; contudo, em períodos mais prolongados existe uma degeneração da unidade neuromuscular com consequente fibrose tecidual, o que pode comprometer o resultado da AHF. ${ }^{11,18,21}$

AAHF clássica é uma técnica confiável que propicia resultados constantes e satisfatórios na reanimação da face paralisada, sendo usada desde o início do século para essa finalidade. ${ }^{2,7,11}$ AAHF é realizada por meio da junção da parte proximal do nervo hipoglosso à porção distal do nervo facial ao nível do forame estilo-mastóideo com suturas não absorvíveis de reparo (fio 10/0) e cola de fibrina. ${ }^{15,16}$ A recuperação ocorre cerca de 6 a 12 meses após o procedimento, atingindo bons resultados (HB II ou III) em $66 \%$ a $79 \%$ dos casos. ${ }^{10,11,15,16}$ As grandes desvantagens do procedimento são a hemiatrofia da língua e a presença de sincinesias, observadas em até $80 \%$ dos pacientes. $2,5,7,10,11,15,17,21$

A incapacidade funcional ocasionada pela disfunção de metade da língua, notadamente nos pacientes portadores de deficiências concomitantes dos nervos cranianos baixos, levou ao desenvolvimento de técnicas que poupassem a função da língua. ${ }^{1,2,4,10,17} \mathrm{Em} 1991$, por meio de um refinamento de técnicas anteriormente utilizadas, May ${ }^{12}$ desenvolveu um procedimento em que se utiliza parte do nervo hipoglosso (50\%) para reanimação da face. ${ }^{12}$ Dessa forma, a metade do nervo hipoglosso é suturada ao nervo facial extracraniano com interposição de um único enxerto (jump graft). ${ }^{4,12}$ É uma técnica que promove resultados consistentes e duráveis, ${ }^{4,5,11}$ apresentando recuperação em sete a oito meses. ${ }^{4}$ As limitações são a necessidade de regeneração por intermédio de duas linhas de sutura, e a oclusão do olho geralmente requer uma técnica estática complementar [implante de peso de ouro na pálpebra ou suspensão com fáscia lata (fascial sling)]., ${ }^{3,4,11}$

Para suplantar essas dificuldades, outro método de reanimação foi desenvolvido, em que por meio de uma abordagem transmastóidea, o nervo facial é seccionado ao nível do joelho externo e rodado inferiormente na direção do nervo hipoglosso. A metade deste é suturada ao nervo facial de forma término-lateral. ${ }^{1,3,4,17}$ A técnica de AHF intrapetrosa término-lateral demonstra uma reinervação relativamente rápida com início tão precoce como dois meses, atingindo resultados no mínimo semelhantes aos das anteriormente apresentadas, todavia não causa atrofia da língua nem tampouco exige a necessidade de técnica estática complementar. ${ }^{1,3,4,17}$ Pode ser usada até mesmo em pacientes referidos tardiamente para tratamento, tendo em vista a rapidez da regeneração, mas a segurança do procedimento deve ser testada numa base maior de pacientes. ${ }^{4}$

\section{Perda da continuidade extracraniana do nervo facial}

As abordagens às lesões extracranianas do nervo facial podem ser agrupadas em cinco tipos de procedimentos: o reparo direto ou com interposição de enxertos; a transferência nervosa transfacial (cross-facial nerve graft); as transferências musculares microneurovasculares; as transferências musculares regionais e os procedimentos estáticos de suporte. - $^{7-9,13,18,19,21,22}$ 
O reparo nervoso direto ou com interposição de enxertos pode ser feito imediatamente após o reconhecimento da lesão do nervo facial extracraniano ou dentro dos primeiros 30 dias, tendo em vista que os resultados tardios são desanimadores. ${ }^{7,18} \mathrm{O}$ enxerto da porção extratemporal do nervo facial tem sido realizado com sucesso, notadamente seguindo-se a cirurgia de glândula parótida, até mesmo após radioterapia. ${ }^{7}$ É o método que apresenta os melhores resultados nesse grupo, entretanto demanda 6 a 12 meses para recuperação, o que poderia limitar seu uso em pacientes com sobrevida curta, uma vez que o tempo necessário para o sucesso pode não justificar o procedimento. ${ }^{7,18}$

Em alguns casos, a lesão do nervo facial é grave o suficiente para não permitir o reparo, sendo o enxerto nervoso transfacial uma possibilidade terapêutica. ${ }^{7,19}$ Essa técnica consiste na anastomose entre o nervo facial normal e o paralisado com enxertos nervosos transfaciais subcutâneos. ${ }^{7}$ Quando tem sucesso, a técnica permite a aquisição de movimentos simétricos, particularmente na região central da face. ${ }^{7}$ É um procedimento de grande exigência técnica, apresentando resultados inconsistentes, especialmente em lesões tardias (mais de seis meses), em que atrofia muscular é presumida. ${ }^{7,8}$ $\mathrm{O}$ procedimento isolado tem aplicabilidade limitada, atingindo os melhores resultados quando associado à transferência muscular microneurovascular., ${ }^{7,8}$

O procedimento combinado em dois estágios, enxerto nervoso transfacial e transferência microneurovascular, oferece a melhor perspectiva de recuperação do tônus muscular, assim como da expressão voluntária em paralisias faciais de longa data. ${ }^{7-9} \mathrm{~A}$ grande desvantagem é o tempo necessário para os resultados aparecerem, cerca de 18 a 24 meses. ${ }^{9}$ Por outro lado, o procedimento em estágio único evita a necessidade de enxerto transfacial e permite uma recuperação mais rápida (dentro de seis meses). ${ }^{8,9}$ Contudo, exige a realização de uma incisão na face do lado normal, no sulco nasolabial., ${ }^{8,9}$ Os músculos grácil e latíssimo do dorso são comumente utilizados para a transferência microneurovascular, ${ }^{7-9,19}$ alcançando resultados considerados bons ou satisfatórios em $90 \%$ dos pacientes. ${ }^{9}$

A transferência muscular regional mostra-se de grande valia nos pacientes em que a paralisia facial não pode ser tratada pela restauração do sistema neuromuscular. ${ }^{71}$ Tem como indicações principais a paralisia facial de longa data e a utilização como método complementar à reanimação primária com enxertos nervosos ou até transferência microneurovascular. ${ }^{7-9,18}$ Os músculos temporal e masseter são os mais utilizados para reanimação do andar inferior da face e eventualmente da pálpebra. ${ }^{7-9,18,21}$ Apresenta como desvantagens a falta de movimentos espontâneos, a disfunção crônica da articulação temporomandibular e a massa sobre o arco zigomático. ${ }^{21} \mathrm{~A}$ ressecção do arco zigomático e a utilização de parte do músculo temporal podem suplantar algumas dessas limitações. ${ }^{18}$

Embora a reconstrução dinâmica seja preferida para o tratamento da paralisia facial completa, os procedimentos estáticos podem ser muito úteis como métodos adjuvantes na melhoria dos resultados cosméticos e funcionais. ${ }^{13} \mathrm{O}$ tratamento cirúrgico dos pacientes com fechamento incompleto do olho com esforço físico máximo inclui o implante de peso de ouro ou platina na pálpebra superior, o lift da sobrancelha, bem como a utilização de suspensão com fáscia lata e blefaroplastia. ${ }^{6,7,13,21}$ Naqueles pacientes sem sorriso significativo, a suspensão do lábio superior e do sulco nasolabial, o lift facial assimétrico, o apoio estático com telas de Marlex, GoreTex ou derme acelular podem ser usados com bons resultados para correção da assimetria médio-facial e perioral. ${ }^{7,13,21}$ Adicionalmente, a toxina botulínica tem um papel importante, especialmente no pós-operatório, para o tratamento da musculatura com sincinesia ou hiperfuncionante. ${ }^{6,21}$ Cabe ressaltar o papel benéfico das terapias estáticas não somente para a compensação de falha nas técnicas dinâmicas, como também precocemente durante o período de observação para recuperação funcional de paralisias faciais reversíveis. ${ }^{6}{ }^{13}$ Nesses casos, o implante palpebral pode ser retirado eletivamente a posteriori caso haja recuperação. ${ }^{6,13}$

\section{Conclusões}

A reanimação da face paralisada permanece um grande desafio, apresentando resultados satisfatórios estéticos e funcionais quando da utilização de técnicas cirúrgicas apropriadas. Atualmente, a grande variedade de técnicas revela o caráter multidisciplinar da reconstrução no âmbito de várias especialidades médicas, como neurocirurgia, cirurgia plástica, otorrinolaringologia e cirurgia de cabeça e pescoço. A reabilitação precoce deve ser considerada em pacientes com lesão estabelecida do nervo facial. Por outro lado, a preservação anatômica ou a ausência de recuperação funcional após reparo direto ou enxerto demandam um período mínimo de 12 meses para recuperação antes da decisão pelo tratamento cirúrgico. As técnicas estáticas podem ser utilizadas precocemente em associação às técnicas dinâmicas, notadamente o implante palpebral, tendo em vista a reversibilidade do método. 


\section{Referências}

1. Asaoka K, Sawamura Y, Nagashima M, Fukushima T. Surgical anatomy for direct hypoglossal-facial nerve side-to-end "anastomosis". J Neurosurg. 1999;91:268-75.

2. Cusimano MD, Sekhar L. Partial hypoglossal to facial nerve anastomosis for reinnervation of the paralyzed face in patients with lower cranial nerve palsies: technical note. Neurosurgery. 1994;35:532-3.

3. Darrouzet V, Guerin J, Bebear JP. New technique of side-toend hypoglossal-facial nerve attachment with translocation of the infratemporal facial nerve. J Neurosurg. 1999;90:27-34.

4. Ferraresi S, Garozzo D, Migliorini V, Buffatti P. End-to-side intrapetrous hypoglossal-facial anastomosis for reanimation of the face. Technical note. J Neurosurg. 2006;104:457-60.

5. Flores LP. Surgical results of the hypoglossal-facial nerve jump graft technique. Acta Neurochir (Wien). 2007;149: 1205-10.

6. Hadlock TA, Greenfield LJ, Wernick-Robinson M, Cheney ML. Multimodality approach to management of the paralyzed face. Laryngoscope. 2006;116:1385-9.

7. Hoffman WY. Reanimation of the paralyzed face. Otolaryngol Clin North Am. 1992;25:649-67.

8. Kumar PA. Cross-face reanimation of the paralysed face, with a single stage microneurovascular gracilis transfer without nerve graft: a preliminary report. $\mathrm{Br} \mathrm{J}$ Plast Surg. 1995;48:83-6.

9. Kumar PA, Hassan KM. Cross-face nerve graft with freemuscle transfer for reanimation of the paralyzed face: a comparative study of the single-stage and two-stage procedures. Plast Reconstr Surg. 2002;109:451-62.

10. Malik TH, Kelly G, Ahmed A, Saeed SR, Ramsden RT. A comparison of surgical techniques used in dynamic reanimation of the paralyzed face. Otol Neurotol. 2005;26:284-91.

11. Manni JJ, Beurskens $\mathrm{CH}$, van de Velde C, Stokroos RJ. Reanimation of the paralyzed face by indirect hypoglossalfacial nerve anastomosis. Am J Surg. 2001;182:268-73.

12. May M, Sobol SM, Mester SJ. Hypoglossal-facial nerve interpositional-jump graft for facial reanimation without tongue atrophy. Otolaryngol Head Neck Surg. 1991;104:818-25.

13. Moser G, Oberascher G. Reanimation of the paralyzed face with new gold weight implants and Goretex soft- tissue patches. Eur Arch Otorhinolaryngol. 1997;254(Supp 1):S76-8

14. Samii M, Koerbel A, Safavi-Abbasi S, Di Rocco F, Samii A, Gharabaghi A. Using an end-to-side interposed sural nerve graft for facial nerve reinforcement after vestibular schwannoma resection. Technical note. J Neurosurg. 2006;105:920-3.

15. Samii M, Matthies C. Indication, technique and results of facial nerve reconstruction. Acta Neurochir (Wien). 1994;130:125-39

16. Samii M, Matthies C. Management of 1000 vestibular schwannomas (acoustic neuromas): the facial nerve preservation and restitution of function. Neurosurgery. 1997;40:684-95.

17. Sawamura Y, Abe H. Hypoglossal-facial nerve side-to-end anastomosis for preservation of hypoglossal function: results of delayed treatment with a new technique. J Neurosurg. 1997;86:203-6.

18. Sobol SM, May M, Mester S. Early facial reanimation following radical parotid and temporal bone tumor resections. Am J Surg. 1980;160:382-6.

19. Takushima A, Harii K, Asato H, Ueda K, Yamada A. Neurovascular free-muscle transfer for the treatment of established facial paralysis following ablative surgery in the parotid region. Plast Reconstr Surg. 2004;113:1563-72.

20. Tatagiba M, Acioly MA. Vestibular schwannoma: Current state-of-the-art. In: Ramina R, Pires Aguiar PH, Tatagiba M, editors. Samii's Essentials in Neurosurgery. 1.ed. Stuttgart: Springer; 2008. p.175-88.

21. Tate JR, Tollefson TT. Advances in facial reanimation. Curr Opin Otolaryngol Head Neck Surg. 2006;14:242-8.

22. Winslow CP, Wang TD, Wax MK. Static reanimation of the paralyzed face with an acellular dermal allograft sling. Arch Facial Plast Surg. 2001;3:55-7.

Original recebido em outubro de 2008

Aceito para publicação em junho de 2009

\section{Endereço para correspondência}

Marcus André Acioly

Boulevard vinte e oito de setembro, 77, $4^{\circ}$ andar-Neurocirurgia

20551-030 - Rio de Janeiro, RJ

E mail: marcusacioly@yahoo.com.br 